\title{
AN ALGEBRA OF DISTRIBUTIONS ON AN OPEN INTERVAL
}

\author{
BY
}

\author{
HARRIS S. SHULTZ
}

\begin{abstract}
Let $(a, b)$ be any open subinterval of the reals which contains the origin and let denote the family of all distributions on $(a, b)$ which are regular in some interval $(\epsilon, 0)$, where $\epsilon<0$. Then $\dot{B}$ is a commutative algebra: Multiplication is defined so that, when restricted to those distributions on $(a, b)$ whose supports are contained in $[0, b)$, it is ordinary convolution. Also, $\mathscr{B}$ can be injected into an algebra of operators; this family of operators is a sequentially complete locally convex space. Since it preserves multiplication, this injection serves as a generalization (there are no growth restrictions) of the two-sided Laplace transformation.
\end{abstract}

In [6] there is introduced a new algebra $B$ of distributions on $(-\infty, \infty)$, closed under convolution and containing the space of distributions having support in $[0, \infty)$ as well as all locally integrable functions. No growth or support restrictions are placed on the elements of $B$. There is also defined a one-to-one transformation of $B$ into a commutative algebra of operators (somewhat analogous to the Fourier transformation). In the present article we generalize these results in obtaining a space $B$ of distributions on $\Omega$, where $\Omega$ is any open subinterval of the reals which contains the origin. A distribution $F$ on $\Omega$ belongs to $B$ if and only if $F$ is regular in some interval $(\epsilon, 0)$, where $\epsilon<0$. Convolution is defined and $B$ is shown to be closed under this operation. It is also shown that the algebra $\mathscr{A}$ into which $B$ can be injected is a sequentially complete locally convex space in which convergence is defined simply in terms of the ordinary pointwise convergence of functions.

0. Preliminaries. Throughout we assume $-\infty \leq a<0<b \leq \infty$ and set $\Omega=$ $(a, b)$. We define $L$ to be the space of all the complex-valued functions which are Lebesgue integrable on each compact subinterval of $\Omega$. We denote by $L_{+}$(respectively, $L_{-}$) the subspace consisting of those elements of $L$ which vanish on $(a, 0)$ (respectively, $(0, b)$ ). If $f$ and $g$ belong to $L$ then the function $f \wedge g$ defined by the equation

$$
f \wedge g(t)=\int_{0}^{t} f(t-u) g(u) d u \quad(t \in \Omega)
$$

Presented to the Society, January 19, 1972; received by the editors June 10, 1971. AMS 1970 subject classifications. Primary 44A40, 44A35, 42A76; Secondary 46F99. Key words and phrases. Generalized functions, operational calculus, Schwartz distributions, two-sided Laplace transformation, Fourier transformation. 
also belongs to $L$, moreover, if we identify functions which are equal almost everywhere on $\Omega$ then

$$
f \wedge g=g \wedge f
$$

(see [4]). For any $f$ in $L$ we define

$$
f_{+}(t)=\left\{\begin{array}{ll}
f(t), & 0 \leq t<b, \\
0, & t<0,
\end{array} \text { and } f_{-}(t)= \begin{cases}0, & t \geq 0, \\
f(t), & a<t<0 .\end{cases}\right.
$$

If $\Omega_{0}$ is an open subinterval of the reals we denote by $\mathscr{D}\left(\Omega_{0}\right)$ the space of complex-valued infinitely differentiable functions defined on the reals which vanish outside of a compact subset of $\Omega_{0}$. If $\phi \in \mathscr{D}\left(\Omega_{0}\right)$ we define the support of $\phi$, denoted supp $\phi$, to be the closure of the set $\{t: \phi(t) \neq 0\}$. Then supp $\phi \subset \Omega_{0}$ for all $\phi$ in $\mathscr{D}\left(\Omega_{0}\right)$.

As usual, the dual of $\mathscr{D}\left(\Omega_{0}\right)$, that is, the space of distributions on $\Omega_{0}$, is denoted by $\mathscr{D}^{\prime}\left(\Omega_{0}\right)$. If $R$ belongs to $\mathscr{D}^{\prime}\left(\Omega_{0}\right)$ and $\phi$ belongs to $\mathscr{D}\left(\Omega_{0}\right)$ the scalar which $R$ assigns to $\phi$ will be written $\langle R(x), \phi(x)\rangle$. If $f$ belongs to the family of locally integrable functions on $\Omega_{0}$ and $m$ is a nonnegative integer we shall write $\partial^{m} f$ for the element of $\mathscr{D}^{\prime}\left(\Omega_{0}\right)$ defined by

$$
\left\langle\partial^{m} f(x), \phi(x)\right\rangle=(-1)^{m} \int_{\Omega_{0}} f(x) \phi^{(m)}(x) d x \quad\left(\phi \in \mathscr{D}\left(\Omega_{0}\right)\right) .
$$

In particular, $\partial^{0} f$ is the regular distribution corresponding to the function $f$. The support of a distribution $R$ on $\Omega_{0}$ (denoted supp $R$ ) is defined to be the complement with respect to $\Omega_{0}$ of the largest open set on which $R$ vanishes.

1. The algebra $\mathscr{B}$. We denote by $\mathscr{T}_{b}^{\prime}$ the space of elements in $\mathscr{D}^{\prime}((-\infty, b))$ having support in $[0, b)$. We denote by $\mathscr{D}_{a}^{\prime}$ the space of elements in $\mathscr{D}^{\prime}((a, \infty))$ having support in $(a, 0]$.

1.01. Definition. Suppose $\left\{b_{n}\right\}$ and $\left\{a_{n}\right\}$ are sequences of real numbers, that $\left\{J_{n}\right\}$ and $\left\{K_{n}\right\}$ are sequences of nonnegative integers and that $\left\{F_{n}\right\}$ and $\left\{G_{n}\right\}$ are sequences in $L$. If the ordered pair $(R, S)$ belongs to the cartesian product $\mathscr{D}_{b}^{\prime} \times \mathscr{D}_{a}^{\prime}$ we say that the sequence $\left\{\left(F_{n}, b_{n}, J_{n}, G_{n}, a_{n}, K_{n}\right)\right\}$ belongs to $\Sigma_{R, S}$ if (1.01.1) $a \leftarrow \cdots<a_{2}<a_{1}<a_{0}=0=b_{0}<b_{1}<b_{2}<\cdots \rightarrow b$;

(1.01.2) $F_{n}$ vanishes on $\left(-\infty, b_{n}\right)$ and $G_{n}$ vanishes on $\left(a_{n}, \infty\right)$;

(1.01.3) $R^{n}=\sum_{n=0}^{\infty} \partial^{J_{n}} F_{n}$ and $S=\sum_{n=0}^{\infty}{ }^{n}{ }^{K}{ }^{n} G_{n}$.

1.02. Theorem. Given any $(R, S)$ in $\mathscr{D}_{b}^{\prime} \times \mathscr{D}_{a}^{\prime}$ and any sequences $\left\{b_{n}\right\}$ and $\left\{a_{n}\right\}$ satisfying (1.01.1) there exists an element $\left\{\left(F_{n}, b_{n}, J_{n}, G_{n}, a_{n}, K_{n}\right)\right\}$ of $\Sigma_{R, S}$ 
Proof. By $[8,2.17]$ there exists a sequence $\left\{F_{n}\right\}$ in $L$ such that the equation

$$
R=\sum_{n=0}^{\infty} \partial^{J} F_{n}
$$

holds for some sequence $\left\{J_{n}\right\}$ of nonnegative integers. We define an element $T$ of $D^{\prime}((-\infty,-a))$ as follows:

$$
\langle T(x), \phi(x)\rangle=\langle S(x), \phi(-x)\rangle \quad(\phi \in \mathfrak{D}((-b,-a))) .
$$

Then, since supp $S \subset(a, 0]$, the distribution $T$ has support contained in $[0,-a)$. Since $0=-a_{0}<-a_{1}<\cdots<-a$ we may infer from $[8,2.17]$ the existence of a sequence $\left\{H_{n}\right\}$ in $L^{\text {loc }}((-\infty,-a))$ such that $H_{n}$ vanishes on $\left(-\infty,-a_{n}\right)$ and such that the equation

$$
T=\sum_{n=0}^{\infty} \partial^{K}{ }^{n} H_{n}
$$

holds for some sequence $\left\{K_{n}\right\}$ of nonnegative integers. If we define

$$
G_{n}(x)=(-1)^{K}{ }^{n} H(-x)
$$

then $G_{n}$ vanishes on $\left(a_{n}, \infty\right)$ and we may combine (1) and (2) to obtain

$$
S=\sum_{n=0}^{\infty} \partial^{K} G_{n}
$$

Therefore, $\left\{\left(F_{n}, b_{n}, J_{n}, G_{n}, a_{n}, K_{n}\right)\right\} \in \Sigma_{R, s}$.

1.03. Definition. For each $\phi$ in $\mathcal{D}((-\infty, b))$ we define $[\phi]^{+}$to be the family of infinitely differentiable functions $\lambda$ on the reals such that $\lambda$ is equal to 1 on a neighborhood of $[0, \infty)$ and vanishes on some interval $\left(-\infty, a^{\prime}\right)$, where supp $\phi \subset$ $\left(-\infty, a^{\prime}+b\right)$. For each $\phi$ in $\mathcal{D}((a, \infty))$ we define $[\phi]^{-}$to be the family of infinitely differentiable functions $\mu$ on the reals such that $\mu$ is equal to 1 on a neighborhood of $(-\infty, 0]$ and vanishes on some interval $\left(b^{\prime}, \infty\right)$, where supp $\phi \subset\left(a+b^{\prime}, \infty\right)$.

1.04. Theorem. Suppose $(r, s)$ and $(R, S)$ belong to $\mathscr{D}_{b}^{\prime} \times \mathscr{D}_{a}^{\prime}$. If $\left\{\left(f_{n}, b_{n}, j_{n}, g_{n}, a_{n}, k_{n}\right)\right\}$ belongs to $\Sigma_{r, s}$ and $\left\{\left(F_{n}, b_{n}, J_{n}, G_{n}, a_{n}, K_{n}\right)\right\}$ belongs to $\Sigma_{R, s}$, then for any $\phi$ in $\mathcal{D}((-\infty, b))$ the equation

$$
\langle r(y),\langle R(x), \lambda(y) \phi(x+y)\rangle\rangle=\lim _{N \rightarrow \infty} \sum_{m=0}^{N} \sum_{n=0}^{N}\left\langle\partial^{j} m^{+J}\left(f_{m} \wedge F_{n}\right)(x), \phi(x)\right\rangle
$$

bolds for all $\lambda$ in $[\phi]^{+}$, and for any $\phi$ in $\mathfrak{I}((a, \infty))$ the equation 


$$
-\langle(s(y),\langle S(x), \mu(y) \phi(x-y)\rangle\rangle
$$

$$
=\lim _{N \rightarrow \infty} \sum_{n=0}^{N} \sum_{n=0}^{N}\left\langle\partial^{k}{ }^{+K}{ }_{n}\left(g_{m} \wedge G_{n}\right)(x), \phi(x)\right\rangle
$$

bolds for all $\mu$ in $[\phi]^{-}$.

Proof. Suppose $\phi \in \mathscr{D}((-\infty, b))$ and $\lambda \in[\phi]^{+}$. There exist numbers $\beta$ and $a^{\prime}$ such that

$$
\operatorname{supp} \phi \subset(-\infty, \beta] \subset\left(-\infty, a^{\prime}+b\right)
$$

and such that $\lambda$ vanishes on $\left(-\infty, a^{\prime}\right)$. For any $y$ the function $x \mapsto \lambda(y) \phi(x+y)$ is infinitely differentiable. From (1) it follows that its support is contained in $(-\infty, \beta-y]$. Thus, for $y \geq a^{\prime}$, its support is contained in $\left(-\infty, \beta-a^{\prime}\right]$ and therefore in $(-\infty, b)$. And, for $y<a^{\prime}$, it vanishes identically (since $\left.\lambda(y)=0\right)$. Consequently, the function $x \mapsto \lambda(y) \phi(x+y)$ belongs to $\mathscr{D}((-\infty, b))$ and has support in $\left(-\infty, \beta-a^{\prime}\right]$ for all $y$. There exists $N$ such that $b_{N+1}>\beta-a^{\prime}$ and therefore

$$
\langle R(x), \lambda(y) \phi(x+y)\rangle=\sum_{n=0}^{\infty}(-1)^{J} n \int_{0}^{b} F_{n}(x) \lambda(y) \phi^{\left(J_{n}\right)}(x+y) d x
$$

$$
=\sum_{n=0}^{N}(-1)^{J} n \int_{0}^{b} F_{n}(x) \lambda(y) \phi^{\left(J_{n}\right)}(x+y) d x
$$

for all $y$ (recall that $F_{n}$ vanishes on $\left(-\infty, b_{n}\right)$ ). From $(2)$ and $[2,250]$ it follows that the function

$$
y \mapsto\langle R(x), \lambda(y) \phi(x+y)\rangle
$$

is infinitely differentiable. From (1) comes the equality

$$
\langle R(x), \lambda(y) \phi(x+y)\rangle=0 \quad(\text { all } y>\beta)
$$

(recall supp $R \subset[0, b)$ ). And

$$
\langle R(x), \lambda(y) \phi(x+y)\rangle=0 \quad\left(\text { all } y<a^{\prime}\right)
$$

since $\lambda$ vanishes on $\left(-\infty, a^{\prime}\right)$. Consequently, the function (3) belongs to $\mathscr{D}((-\infty, b))$. Moreover, we may combine (4) and the inequality $b_{N+1}>\beta$ to obtain

$$
\int_{0}^{b} f_{m}(y)\langle R(x), \lambda(y) \phi(x+y)\rangle d y=0 \quad(\text { all } m>N) .
$$

Therefore, 


$$
\begin{aligned}
& \langle r(y),\langle R(x), \lambda(y) \phi(x+y)\rangle\rangle \\
& \quad=\sum_{m=0}^{N}(-1)^{j} m \int_{0}^{b} f_{m}(y) \frac{d^{j} m}{d y^{j}}\langle R(x), \lambda(y) \phi(x+y)\rangle d y \\
& \quad=\sum_{m=0}^{N} \sum_{n=0}^{N}(-1)^{j}{ }^{+J} n \int_{0}^{b} f_{m}(y)\left(\int_{0}^{b} F_{n}(x) \phi^{\left(j_{m}+J_{n}\right)}(x+y) d x\right) d y ;
\end{aligned}
$$

the last equality is from (2), [2, 250] and the fact that $\lambda=1$ on $[0, \infty)$. We may now use the change of variable $t=x+y$ and $[2,283]$ to obtain

$$
\begin{aligned}
\langle r(y),\langle R(x), \lambda(y) \phi(x+y)\rangle\rangle & \\
= & \sum_{m=0}^{N} \sum_{n=0}^{N}(-1)^{j} m^{+J} n \int_{0}^{b}\left(\int_{y}^{b} f_{m}(y) F_{n}(t-y) \phi^{\left(j_{m}+J_{n}\right)}(t) d t\right) d y \\
= & \sum_{m=0}^{N} \sum_{n=0}^{N}(-1)^{j_{m}+J} n \int_{0}^{b}\left(\int_{0}^{t} f_{m}(y) F_{n}(t-y) d y\right) \phi^{\left(j_{m}+J\right.}{ }^{\prime}(t) d t .
\end{aligned}
$$

We need only observe now that

$$
\int_{0}^{t} f_{m}(y) F_{n}(t-y) d y=0 \quad(0 \leq t \leq \beta)
$$

for $m>N$ and $n>N$ to obtain (1.04.1). Suppose now that $\phi \in \mathscr{D}((a, \infty))$ and $\mu \epsilon$ $[\phi]^{-}$. There exist numbers $\alpha$ and $b^{\prime}$ such that

$$
\operatorname{supp} \phi \subset[a, \infty) \subset\left(a+b^{\prime}, \infty\right)
$$

and such that $\mu$ vanishes on $\left(b^{\prime}, \infty\right)$. For any $y$ the function $x \mapsto \mu(y) \phi(x+y)$ is infinitely differentiable. From (5) it follows that its support is contained in $[a-y, \infty)$. Thus, for $y \leq b^{\prime}$, its support is contained in $\left[a-b^{\prime}, \infty\right)$ and therefore in $(a, \infty)$. And, for $y>b^{\prime}$, it vanishes identically (since $\left.\mu(y)=0\right)$. Consequently, the function $x \mapsto \mu(y) \phi(x+y)$ belongs to $\mathcal{D}((a, \infty))$ and has support in $\left[a-b^{\prime}, \infty\right)$ for all $y$. There exists $N$ such that $a_{N+1}<a-b^{\prime}$ and therefore

$$
\langle S(x), \mu(y) \phi(x+y)\rangle=\sum_{n=0}^{\infty}(-1)^{K} \int_{a}^{0} G_{n}(x) \mu(y) \phi^{\left(K_{n}\right)}(x+y) d x
$$

$$
=\sum_{n=0}^{N}(-1)^{K} \int_{a}^{0} G_{n}(x) \mu(y) \phi^{\left(K_{n}\right)}(x+y) d x
$$

for all $y$ (recall that $G_{n}$ vanishes on $\left(a_{n}, \infty\right)$ ). From $(6)$ and $[2,250]$ it follows that the function 


$$
y \mapsto\langle S(x), \mu(y) \phi(x+y)\rangle
$$

is infinitely differentiable. From (5) comes the equality

$$
\langle S(x), \mu(y) \phi(x+y)\rangle=0 \quad(\text { all } y<\alpha)
$$

(recall $\operatorname{supp} S \subset(a, 0])$. And

$$
\langle S(x), \mu(y) \phi(x+y)\rangle=0 \quad\left(\text { all } y>b^{\prime}\right)
$$

since $\mu$ vanishes on $\left(b^{\prime}, \infty\right)$. Consequently, the function (7) belongs to $\mathscr{D}((a, \infty))$. Moreover, we may combine (8) and the inequality $a_{N+1}<\alpha$ to obtain

$$
\int_{a}^{0} g_{m}(y)\langle S(x), \mu(y) \phi(x+y)\rangle d y=0 \quad(\text { all } m>N) .
$$

Therefore,

$$
\begin{aligned}
& \langle s(y),\langle S(x), \mu(y) \phi(x+y)\rangle\rangle \\
& \quad=\sum_{m=0}^{N}(-1)^{k} m \int_{a}^{0} g_{m}(y) \frac{d^{k} m}{d y^{j} m}\langle S(x), \mu(y) \phi(x+y)\rangle d y \\
& \quad=\sum_{m=0}^{N} \sum_{n=0}^{N}(-1)^{k_{m}+K_{n}} \int_{a}^{0} g_{m}(y)\left(\int_{a}^{0} G_{n}(x) \phi^{\left(k_{m}+K_{n}\right)}(x+y) d x\right) d y
\end{aligned}
$$

the last equality is from (5), $[2,250]$ and the fact that $\mu=1$ on $(-\infty, 0]$. We may now use the change of variable $t=x+y$ and $[2,283]$ to obtain

$$
\begin{aligned}
&\langle s(y),\langle S(x), \mu(y) \phi(x+y)\rangle\rangle \\
& \quad=\sum_{m=0}^{N} \cdot \sum_{n=0}^{N}(-1)^{k_{m}+K} n \int_{a}^{0}\left(\int_{a}^{y} g_{m}(\dot{y}) G_{n}(t-y) \phi^{\left(k_{m}+K_{n}\right)}(t) d t\right) d y \\
&=\sum_{m=0}^{N} \sum_{n=0}^{N}(-1)^{k_{m}+K} n \int_{a}^{0}\left(\int_{t}^{0} g_{m}(y) G_{n}(t-y) d y\right) \phi^{\left(k_{m}+K_{n}\right)}(t) d t .
\end{aligned}
$$

We need only observe now that

$$
\int_{t}^{0} g_{m}(y) G_{n}(t-y) d y=0 \quad(\alpha \leq t \leq 0)
$$

for $m>N$ and $n>N$ and that

$$
-\int_{t}^{0} g_{m}(y) G_{n}(t-y) d y=g_{n} \wedge G_{n}(t)
$$

to obtain (1.04.2). 
1.05. Corollary. Suppose that $(r, s)$ and $(R, S)$ belong to $\mathfrak{D}_{b}^{\prime} \times \mathscr{D}_{a}^{\prime}$. For any $\phi$ in $D((-\infty, b))$ the family

$$
\left\{\langle r(y),\langle R(x), \lambda(y) \phi(x+y)\rangle): \lambda \in[\phi]^{+}\right\}
$$

contains a unique element, which will be denoted by $\langle r * R(x), \phi(x)\rangle$. For any $\phi$ in $\mathscr{D}((a, \infty))$ the family

$$
\left\{\langle s(y),\langle S(x), \mu(y) \phi(x+y)\rangle): \mu \in[\phi]^{-}\right\}
$$

contains a unique element, which will be denoted by $\langle s * S(x), \phi(x)\rangle$.

1.06. Definition. Let $(r, s)$ and $(R, s)$ belong to $\mathscr{D}_{b}^{\prime} \times \mathscr{D}_{a}^{\prime}$. We denote by $r * R$ the functional that assigns to any $\phi$ in $\mathcal{D}((-\infty, b))$ the number $\langle r * R(x), \phi(x)\rangle$; we denote by $s * S$ the functional that assigns to any $\phi$ in $\mathcal{D}((a, \infty))$ the number $\langle s * S(x), \phi(x)\rangle$.

1.07. Remark. It follows from 1.04 and (0.02) that $r * R=R * r$ and $s * S=$ $S * s$,

1.08. Corollary. If $(r, s)$ and $(R, S)$ belong to $\mathscr{D}_{b}^{\prime} \times \mathscr{D}_{a}^{\prime}$ then $(r * R, s * S)$ belongs to $\mathfrak{T}_{b}^{\prime} \times \mathfrak{T}_{a}^{\prime}$.

Proof. It follows from (1.04.1) and the sequential completeness of $\mathscr{D}^{\prime}((-\infty, b))$ (see [1, Proposition 2, p. 315]) that $r * R$ belongs to $\mathscr{D}^{\prime}((-\infty, b))$. Moreover, since $r * R$ is the limit of a sequence of distributions on $(-\infty, b)$ all having support in $[0, b)$, it too must have support in $[0, b)$, i.e. $r * R \in \mathfrak{D}_{b}^{\prime}$. Similarly, $s * S \in$ $\mathfrak{D}_{a}^{\prime}$.

1.09. Remark. If $f$ belongs to $L$ then $\partial^{0} f_{+} \in \mathscr{D}_{b}^{\prime}$ and $\partial^{0} f_{-} \in \mathscr{D}_{a}^{\prime}$. We may deduce from 1.01 and 1.04 that the equations $\partial^{0} f_{+}{ }^{*} \partial^{0} g_{+}=\partial^{0}\left(f_{+} \wedge^{a} g_{+}\right)$and $-\partial^{0} f_{-} * \partial^{0} g_{-}=\partial^{0}\left(f_{-} \wedge g_{-}\right)$hold for all $f$ and $g$ in $L$.

1.10. Definition. We denote by $B$ _ the linear subspace consisting of those elements of $\mathscr{D}_{a}^{\prime}$ which are regular in a neighborhood of the origin.

1.11. Remark. Thus $S \in B_{-}$if and only if $S=\partial^{0} f+T$ where $f \in L_{\text {- }}$ and $T \in \mathscr{D}_{a}^{\prime}$ with supp $T \subset(a, 0)$. In particular, $\partial^{0} f_{-} \in B_{-}$for all $f \in L$.

1.12. Lemma. Suppose $(R, S)$ and $\left(R_{1}, S_{1}\right)$ belong to $D_{b}^{\prime} \times B_{\text {. }}$. If the elements $R+S$ and $R_{1}+S_{1}$ of $\mathcal{D}^{\prime}(\Omega)$ are equal then $R=R_{1}$ and $S=S_{1}$.

Proof. Since $S$ and $S_{1}$ both vanish on $(0, b)$ we have

$$
S=S_{1} \text { on }(0, b) \text {. }
$$

Since $R$ and $R_{1}$ both vanish on $(a, 0)$ it follows from $R+S=R_{1}+S_{1}$ that

$$
S=S_{1} \text { on }(a, 0)
$$


Now, there exists $\epsilon>0$ and elements $f$ and $f_{1}$ of $L_{-}$such that $S=\partial^{0} f$ and $S_{1}=$ $\partial^{0} f_{1}$ on $(-\epsilon, \epsilon)$. From (1) and (2) it follows that $\partial^{0} f=\partial^{0} f_{1}$ on $(0, \epsilon)$ and on $(-\epsilon, 0)$. Thus, by $\left[9\right.$, p. 224] we have $f=f_{1}$ almost everywhere on $(-\epsilon, \epsilon)$, from which it follows that $\partial^{0} f=\partial^{0} f_{1}$ on $(-\epsilon, \epsilon)$. Therefore,

$$
S=\partial^{0} f=\partial^{0} f_{1}=S_{1} \text { on }(-\epsilon, \epsilon) .
$$

We may now combine (1), (2) and (3) to conclude that $S=S_{1}$ on $(a, b)$ (see [9, Theorem 24.1]).

1.13. Definition. We denote by $B$ the linear subspace consisting of those elements of $\mathscr{D}^{\prime}(\Omega)$ of the form $R+S$ where $R \in \mathscr{D}_{b}^{\prime}$ and $S \in \mathbb{B}_{-}$.

1.14. Remark. Thus, $F \in \mathscr{B}$ if and only if $F \in \mathscr{D}^{\prime}(\Omega)$ and is regular in some neighborhood $(\epsilon, 0)$, where $a<\epsilon<0$. In particular, $\partial^{0} f \in B$ for all $f \in L$.

1.15. Theorem. If $F$ belongs to $B$ there exists a unique element of $\mathfrak{T}_{b}^{\prime} \times \mathbb{B}$, denoted $\left(F_{+}, F_{-}\right)$, such that $F=F_{+}+F_{-}$.

Proof. Immediate from 1.12.

1.16. Corollary. The mapping $F \mapsto\left(F_{+}, F_{-}\right)$is an isomorphism of $B$ into $\mathfrak{D}_{b}^{\prime} \times \mathfrak{T}_{a}^{\prime}$.

Proof. One may easily verify that the mapping $F \mapsto\left(F_{+}, F_{-}\right)$is linear. The corollary then follows from 1.15 .

1.17. Lemma. If $V$ and $V_{1}$ belong to $\mathfrak{L}_{a}^{\prime}$ with $\operatorname{supp} V_{1} \subset(a, \epsilon]$ for some $\epsilon<$ 0 then supp $V * V_{1} \subset(a, \epsilon]$.

Proof. Let $\phi \in \mathscr{D}((a, \infty))$ and have support in $\left[\epsilon^{\prime}, \infty\right)$, where $\epsilon<\epsilon^{\prime}<0$. Then, for $y<\epsilon^{\prime}-\epsilon$ the function $x \mapsto \phi(x+y)$ has support contained in $(\epsilon, \infty)$. Therefore,

$$
\left\langle V_{1}(x), \mu(y) \phi(x+y)\right\rangle=0 \quad\left(\text { all } \mu \in[\phi]^{-}\right)
$$

for all $y<\epsilon^{\prime}-\epsilon$. Thus, the function $y \longmapsto\left\langle V_{1}(x), \mu(y) \phi(x+y)\right\rangle$ has support contained in $(0, \infty)$ for all $\mu \in[\phi]^{-}$. Since $V$ vanishes on this interval it follows that

$$
\left\langle V * V_{1}(x), \phi(x)\right\rangle=\left\langle V(y),\left\langle V_{1}(x), \mu(y) \phi(x+y)\right\rangle\right\rangle=0
$$

for all $\mu$ in $[\phi]-$. Therefore, $V * V_{1}$ vanishes on $(\epsilon, \infty)$.

1.18. Theorem. If $F$ and $G$ belong to $B$ then $F_{+} * G_{+}-F_{-} * G_{-}$belongs to $B$ with $\left(F_{+} * G_{+}-F_{-} * G_{-}\right)_{-}=-F_{-} * G_{-}$.

Proof. It suffices to show that $F_{-}{ }^{*} G_{-} \in B_{-}$. By 1.11 there exist $f$ and $g$ in $L_{-}$and $T$ and $U$ in $\mathscr{D}_{a}^{\prime}$ such that $F_{-}=\partial^{0} f+T$ and $G_{-}=\partial^{0} g+U$ with 
supp $T \subset(a, \epsilon]$ and supp $U \subset(a, \epsilon]$ for some $\epsilon<0$. Therefore,

$$
F_{-} * G_{-}=\left(\partial^{0} f+T\right) *\left(\partial^{0} g+U\right)=\left(\partial^{0} f\right) *\left(\partial^{0} g\right)+\left(\partial^{0} f\right) * U+T *\left(\partial^{0} g\right)+T * U
$$

From 1.09 and 1.07 it follows that

$$
F_{-} * G_{-}=\partial^{0}(f \wedge g)+\left(\partial^{0} f\right) * U+\left(\partial^{0} g\right) * T+T * U
$$

If we set $S=\left(\partial^{0} f\right) * U+\left(\partial^{0} g\right) * T+T * U$ we may infer from 1.17 that $\operatorname{supp} S \subset$ $(a, \epsilon]$ and therefore $F_{-} * G_{-} \in B_{-}$.

1.19. Definition. If $F$ and $G$ belong to $\mathscr{P}$ we denote the element $F_{+}{ }^{*} G_{+}-$ $F_{-} * G_{-}$of $\mathscr{P}$ by $F \wedge G$.

1.20. Remark. As a consequence of 2.23 , the space $\mathscr{B}$, with multiplication defined by 1.19 , is a commutative algebra.

1.21. Theorem. The equation $\partial^{0}(f \wedge g)=\left(\partial^{0} f\right) \wedge\left(\partial^{0} g\right)$ bolds for all $f$ and $g$ in $L$.

Proof. Since $f \wedge g=f_{+} \wedge g_{+}+f_{-} \wedge g_{-}$we may use 1.09 to obtain

$$
\begin{aligned}
\partial^{0}(f \wedge g) & =\partial^{0}\left(f_{+} \wedge g_{+}\right)+\partial^{0}\left(f_{-} \wedge g_{-}\right)=\partial^{0} f_{+} * \partial^{0} g_{+}-\partial^{0} f_{-} * \partial^{0} g_{-} \\
& =\left(\partial^{0} f\right)_{+} *\left(\partial^{0} g\right)_{+}-\left(\partial^{0} f\right)_{-} *\left(\partial^{0} g\right)_{-}=\left(\partial^{0} f\right) \wedge\left(\partial^{0} g\right) .
\end{aligned}
$$

2. The algebra of operators. Let $W$ be the space of all the complex-valued infinitely differentiable functions $w$ on $\Omega$ such that $w^{(k)}(0)=0$ for $k \geq 0$. In [4] it is shown that $f \wedge w$ belongs to $W$ with

$$
(f \wedge w)^{\prime}=f \wedge w^{\prime}
$$

whenever $f$ belongs to $L$ and $w$ belongs to $W$. We denote by $\langle f\rangle$ the operator which assigns to each $w$ in $W$ the function $f \wedge w$ in $W$. Thus, $\langle f\rangle_{w=f} \wedge w$ (all $w$ in $W$ ). Let $\mathfrak{A}$ be the set of all the operators $A$ mapping $W$ into itself such that

$$
A\left(w_{1} \wedge w_{2}\right)=\left(A w_{1}\right) \wedge w_{2}
$$

for all $w_{1}$ and $w_{2}$ in $W$. We make $\mathfrak{Q}$ into a vector space by defining addition and scalar multiplication in the usual way. We define the product of two operators to be the composition of the operators. Then $\mathscr{Q}$ is a commutative algebra which contains the identity operator $I$ and the differentiation operator $D$; moreover, the mapping $f \mapsto\langle f\rangle$ is a linear injection of $L$ into $\mathscr{Q}$ and

$$
\langle f \wedge g\rangle=\langle f\rangle\langle g\rangle
$$

for all $f$ and $g$ in $L$ (see [4]).

2.04. Theorem. If $\left\{\left(F_{n}, b_{n}, J_{n}, G_{n}, a_{n}, K_{n}\right)\right\}$ belongs to $\Sigma_{R, s}$ for some $(R, S)$ in $\mathscr{D}_{b}^{\prime} \times \mathscr{D}_{a}^{\prime}$ then the equation 


$$
A w(t)=\sum_{n=0}^{\infty}\left(\left(F_{n} \wedge w\right)^{\left(J_{n}\right)}(t)+\left(G_{n} \wedge w\right)^{\left(K_{n}\right)}(t)\right) \quad(t \in \Omega, w \in W)
$$

defines an element of $\mathbb{A}$.

Proof. Let $w \in W$ and $a<\alpha<\beta<b$. Then there exists a positive integer $N$ such that $a_{N+1}<a<\beta<b_{N+1}$. Since $F_{n}$ vanishes on $\left(a, b_{n}\right)$ we may infer that $F_{n} \wedge w$ vanishes on $(a, \beta)$ for all $n>N$; since $G_{n}$ vanishes on $\left(a_{n}, b\right)$ we may infer that $G_{n} \wedge w$ vanishes on $(a, b)$ for all $n>N$. Consequently,

$$
A w(t)=\sum_{n=0}^{N}\left(\left(F_{n} \wedge w\right)^{\left(J_{n}\right)}(t)+\left(G_{n} \wedge w\right)^{\left(K_{n}\right)}(t)\right) \quad(\alpha<t<\beta) .
$$

Since each $F_{n} \wedge w$ and each $G_{n} \wedge w$ is infinitely differentiable on $(\alpha, \beta)$ it follows from (1) that $A w$ is infinitely differentiable on $(\alpha, \beta)$; and, clearly, every derivative of $A w$ vanishes at the origin since the same is true of each term on the right-hand side of (1). Since $(\alpha, \beta)$ was an arbitrary open subinterval of $\Omega$ we may conclude that $A w \in W$. There remains to show that the equation $A\left(w_{1} \wedge w_{2}\right)=$ $\left(A w_{1}\right) \wedge w_{2}$ holds for all $w_{1}$ and $w_{2}$ in $W$. But, using (2.01) and the fact that $\left\langle F_{n}\right\rangle$ and $\left\langle G_{n}\right\rangle$ belong to $\mathscr{A}$ we may deduce that

$$
\begin{aligned}
A\left(w_{1} \wedge w_{2}\right) & =\sum_{n=0}^{\infty}\left(\left(F_{n} \wedge\left(w_{1} \wedge w_{2}\right)\right)^{\left(J_{n}\right)}+\left(G_{n} \wedge\left(w_{1} \wedge w_{2}\right)\right)^{\left(K_{n}\right)}\right) \\
& =\sum_{n=0}^{\infty}\left(\left(\left(F_{n} \wedge w_{1}\right) \wedge w_{2}\right)^{\left(J_{n}\right)}+\left(\left(G_{n} \wedge w_{1} \backslash \wedge w_{2}\right)^{\left(K_{n}\right)}\right)\right. \\
& =\sum_{n=0}^{\infty}\left(\left(F_{n} \wedge w_{1}\right)^{\left(J_{n}\right)} \wedge w_{2}+\left(G_{n} \wedge w_{1}\right)^{\left(K_{n}\right)} \wedge w_{2}\right) \\
& =\left(\sum_{n=0}^{\infty}\left(\left(F_{n} \wedge w_{1}\right)^{\left(J_{n}\right)}+\left(G_{n} \wedge w_{1}\right)^{\left(K_{n}\right)}\right)\right) \wedge w_{2}=\left(A w_{1}\right) \wedge w_{2} .
\end{aligned}
$$

For each $w$ in $W$ and each $t$ in $\Omega$ the equation $\rho_{w, t}(A)=|A w(t)|$ defines a seminorm on the space $\mathfrak{A}$. Let $\mathfrak{Q}$ be endowed with the locally convex topology defined by the family of seminorms $\left\{\rho_{w, t}: t \in \Omega, w \in W\right\}$.

2.05. Remark. If $\left\{A_{n}\right\}$ is a sequence in $\mathbb{Q}$ then $A_{0}=\lim A_{n}$ if and only if $A_{0} w(t)=\lim A_{n} w(t)$ for all $w$ in $W$ and all $t$ in $\Omega$. 
2.06. Definition. If $0 \leq t<b$ we denote by $[t]$ the set of all infinitely differentiable functions $p$ which assume the value 1 on a neighborhood of $[0, \infty)$ and which vanish on some interval $\left(-\infty, \alpha_{p}\right)$, where $t-b<\alpha_{p}$. If $a<t<0$ we let $[t]$ denote the set of all infinitely differentiable functions $q$ which assume the value 1 on a neighborhood of $(-\infty, 0]$ and which vanish on some interval $\left(\beta_{q}, \infty\right)$, where $\beta_{q}<t-a$.

2.07. Definition. If $w$ belongs to $W$ and $0 \leq t<b$ we define

$$
w_{t}(x)= \begin{cases}w(t-x), & t-b<x<t \\ 0, & \text { otherwise }\end{cases}
$$

if $w$ belongs to $W$ and $a<t<0$ we define

$$
w_{t}(x)= \begin{cases}w(t-x), & t<x<t-a \\ 0, & \text { otherwise. }\end{cases}
$$

2.08. Remark. If $w \in W$ and $0 \leq t<b$, the function $w_{t}$ is infinitely differentiable on $(t-b, \infty)$ and vanishes on $(t, \infty)$; thus, if $p \in[t]$, the function $p w_{t}$ (the pointwise product of the functions $p$ and $\left.w_{t}\right)$ belongs to $\mathcal{D}((-\infty, b))$ with supp $p w_{t} \subset(-\infty, t]$. If $w \in W$ and $a<t<0$, the function $w_{t}$ is infinitely differentiable on $(-\infty, t-a)$ and vanishes on $(-\infty, t)$; thus, if $q \in[t]$, the function $q w_{t}$ belongs to $\mathscr{T}((a, \infty))$ with supp $q w_{t} \subset[t, \infty)$.

2.09. Lemma. If $f$ belongs to $L$ and $m$ is a nonnegative integer, the equation

$$
\left\langle\partial^{m} f_{+}(x), p(x) w_{t}(x)\right\rangle=(f \wedge w)^{(m)}(t) \quad(0 \leq t<b)
$$

bolds for any $p$ in $[t]$ and any $w$ in $W$ and the equation

$$
-\left\langle\partial^{m} f_{-}(x), q(x) w_{t}(x)\right\rangle=(f \wedge w)^{(m)}(t) \quad(a<t<0)
$$

bolds for any $q$ in $[t]$ and any $w$ in $W$.

Proof. Let $0 \leq t<b$ and $p \in[t]$. For any $w \in W$,

$$
\left\langle\partial^{m} f_{+}(x), p(x) w_{t}(x)\right\rangle=(-1)^{m} \int_{0}^{b} f(x)\left[p w_{t}\right]^{(m)}(x) d x .
$$

Since $p=1$ on $[0, b)$ and since $w_{t}$ vanishes on $(t, \infty)$ it follows that

$$
\left\langle\partial^{m} f_{+}(x), p(x) w_{t}(x)\right\rangle=(-1)^{m} \int_{0}^{t} f(x)\left[w_{t}\right]^{(m)}(x) d x .
$$


By observing that $(-1)^{m}\left[w_{t}\right]^{(m)}(x)=w^{(m)}(t-x)$ for $x>t-b$, we may use (2.01) to obtain

$$
\left\langle\partial^{m} f_{+}(x), p(x) w_{t}(x)\right\rangle=\int_{0}^{t} f(x) w^{(m)}(t-x) d x=(f \wedge w)^{(m)}(t) .
$$

Now, let $a<t<0$ and $q \in[t]$. For any $w \in W$,

$$
\begin{aligned}
\left\langle\partial^{m} f_{-}(x), q(x) w_{t}(x)\right\rangle & =(-1)^{m} \int_{a}^{0} f(x)\left[q w_{t}\right]^{(m)}(x) d x \\
& =(-1)^{m} \int_{t}^{0} f(x)\left[w_{t}\right]^{(m)}(x) d x=\int_{t}^{0} f(x) w^{(m)}(t-x) d x \\
& =-\int_{0}^{t} f(x) w^{(m)}(t-x) d x=-(f \wedge w)^{(m)}(t) .
\end{aligned}
$$

2.10. Theorem. If $(R, S)$ belongs to $\mathscr{T}_{b}^{\prime} \times \mathscr{D}_{a}^{\prime}$ there exists an element $A$ of Q such that the equation

$$
\left\langle R(x), p(x) w_{t}(x)\right\rangle=A w(t) \quad(0 \leq t<b)
$$

bolds for any $p$ in $[t]$ and any $w$ in $W$ and such that the equation

$$
-\left\langle S(x), q(x) w_{t}(x)\right\rangle=A w(t) \quad(a<t<0)
$$

bolds for any $q$ in $[t]$ and any $w$ in W. If $\left\{\left(F_{n}, b_{n}, J_{n}, G_{n}, a_{n}, K_{n}\right)\right\}$ belongs to $\Sigma_{R, S}$ then

$$
A=\sum_{n=0}^{\infty}\left(D^{J}\left\langle f_{n}\right\rangle+D^{K}\left\langle g_{n}\right\rangle\right)
$$

Proof. Let $\left\{\left(F_{n}, b_{n}, J_{n}, G_{n}, a_{n}, K_{n}\right)\right\} \in \Sigma_{R, s}$. Then, by 2.09,

(1) $\left\langle R(x), p(x) w_{t}(x)\right\rangle=\sum_{n=0}^{\infty}\left\langle\partial^{J} F_{n}(x), p(x) w_{t}(x)\right\rangle=\sum_{n=0}^{\infty}\left(F_{n} \wedge w\right)^{\left(J_{n}\right)}(t)$

for $0 \leq t<b$, any $p \in[t]$ and any $w$ in $W$. Similarly,

(2) $\quad-\left\langle S(x), q(x) w_{t}(x)\right\rangle=\sum_{n=0}^{\infty}-\left\langle\partial^{K}{ }^{K} G_{n}(x), q(x) w_{t}(x)\right\rangle=\sum_{n=0}^{\infty}\left(G_{n} \wedge w\right)^{\left(K_{n}\right)}(t)$

for $a<t<0$, any $q \in[t]$ and any $w$ in $W$. If we define

$$
A w(t)=\sum_{n=0}^{\infty}\left(\left(F_{n} \wedge w\right)^{\left(J_{n}\right)}(t)+\left(G_{n} \wedge w\right)^{\left(K_{n}\right)}(t)\right) \quad(t \in \Omega, w \in W)
$$


then $A \in \mathfrak{Q}$ by 2.04. Moreover, since each $F_{n} \in L_{+}$and each $G_{n} \in L_{-}$we have

$$
A w(t)= \begin{cases}\sum_{n=0}^{\infty}\left(F_{n} \wedge w\right)^{\left(J_{n}\right)}(t) & \text { for } 0 \leq t<b, \\ \sum_{n=0}^{\infty}\left(G_{n} \wedge w\right)^{\left(K_{n}\right)}(t) \text { for } a<t<0,\end{cases}
$$

from which follows the theorem.

2.11. Corollary. Suppose that $(R, S)$ belongs to $\mathscr{D}_{b}^{\prime} \times \mathscr{D}_{a}^{\prime}$ and $w \in W$. If $0 \leq$ $t<b$, the family $\left\{\left\langle R(x), p(x) w_{t}(x)\right\rangle: p \in[t]\right\}$ contains a unique element. If $a<t<$ 0 , the family $\left\{\left\langle S(x), q(x) w_{t}(x)\right\rangle: q \in[t]\right\}$ contains a unique element. If we define

$$
\langle(R, S)\rangle w(t)= \begin{cases}\left\langle R(x), p(x) w_{t}(x)\right\rangle & (p \in[t], 0 \leq t<b) \\ -\left\langle S(x), q(x) w_{t}(x)\right\rangle & (q \in[t], a<t<0)\end{cases}
$$

and denote by $\langle(R, S)\rangle$ the mapping $w \mapsto\langle(R, S)\rangle w$, then $\langle(R, S)\rangle \in \mathbb{Q}$.

2.12. Corollary. If $(R, S) \in \mathfrak{D}_{b}^{\prime} \times \mathscr{D}_{a}^{\prime}$ and $\left\{\left(F_{n}, b_{n}, J_{n}, G_{n}, a_{n}, K_{n}\right)\right\} \in \Sigma_{R, S}$, then

$$
\langle(R, S)\rangle=\sum_{n=0}^{\infty}\left(D^{J}\left\langle F_{n}\right\rangle+D^{K}\left\langle G_{n}\right\rangle\right) .
$$

Proof. Immediate from (2.10.3).

2.13. Corollary. The equation $\left\langle\left(\partial^{m} f_{+}, \partial^{n} f_{-}\right\rangle\right\rangle=D^{m}\left\langle f_{+}\right\rangle+D^{n}\left\langle f_{-}\right\rangle$bolds for all $f$ in $L$ and all nonnegative integers $m$ and $n$.

Proof. Immediate from 2.09.

2.14. Lemma. There exists a sequence $\left\{w_{n}\right\}$ in W such that

$$
A=\lim _{n \rightarrow \infty}\left\langle A w_{n}\right\rangle
$$

for all $A$ in $\mathfrak{Q}$.

Proof. Choose $w_{n}$ in $W$ satisfying

$$
\begin{aligned}
& w_{n} \geq 0 \text { on }(0, b), \\
& w_{n} \leq 0 \text { on }(a, 0), \\
& w_{n}(t)=0 \quad \text { for }|t| \geq 1 / n, \\
& \int_{0}^{b} w_{n}(x) d x=1=-\int_{a}^{0} w_{n}(x) d x
\end{aligned}
$$


(cf. [1, p. 166]). Let $A \in \mathbb{Q}$ and $w \in W$. Then, for $0<t<b$ and $n$ sufficiently large so that $t-1 / n>0$,

$$
(A w) \wedge w_{n}(t)=\int_{t-1 / n}^{t} A w(x) w_{n}(t-x) d x
$$

And, by (3)-(4), we may write

$$
A w(t)=\int_{t-1 / n}^{t} A w(t) w_{n}(t-x) d x
$$

Consequently,

$$
\begin{aligned}
\left|(A w) \wedge w_{n}(t)-A w(t)\right| & =\left|\int_{t-1 / n}^{t}[A w(x)-A w(t)] w_{n}(t-x) d x\right| \\
& \leq \sup _{|x-t| \leq 1 / n}|A w(x)-A w(t)| \int_{t-1 / n}^{t} w_{n}(t-x) d x \\
& =\sup _{|x-t| \leq 1 / n}|A w(x)-A w(t)| .
\end{aligned}
$$

Using (5) and the continuity of $A w$ at $t$ we obtain

$$
A w(t)=\lim _{n \rightarrow \infty}(A w) \wedge w_{n}(t) \quad(0<t<b) .
$$

For $a<t<0$ and $n$ sufficiently large so that $t+1 / n>0$,

$$
(A w) \wedge w_{n}(t)=-\int_{t}^{t+1 / n} A w(x) w_{n}(t-x) d x .
$$

And, by (3)-(4), we may write

$$
A w(t)=-\int_{t}^{t+1 / n} A w(t) w_{n}(t-x) d x
$$

Consequently,

$$
\left|(A w) \wedge w_{n}(t)-A w(t)\right|=\left|-\int_{t}^{t+1 / n}[A w(x)-A w(t)] w_{n}(t-x) d x\right|
$$

$$
\begin{aligned}
& \leq \sup _{|x-t| \leq 1 / n}|A w(x)-A w(t)|\left(-\int_{t}^{t+1 / n} w_{n}(t-x) d x\right) \\
& =\sup _{|x-t| \leq 1 / n}|A w(x)-A w(t)| .
\end{aligned}
$$

Using (7) and the continuity of $A w$ at $t$ we obtain 


$$
A w(t)=\lim _{n \rightarrow \infty}(A w) \wedge w_{n}(t) \quad(a<t<0)
$$

Observing that $(A w) \wedge w_{n}=\left(A w_{n}\right) \wedge w$ and the fact that $\left(A w_{n}\right) \wedge w(0)=0=A w(0)$ we infer from (6) and (8) that $A w(t)=\lim _{n \rightarrow \infty}\left(A w_{n}\right) \wedge w(t)$ (all $t \in \Omega$ ) and therefore that $A=\lim \left\langle A w_{n}\right\rangle$.

2.15. Remark. It follows from 2.14 that each $A$ in $\mathfrak{Q}$ is linear.

2.16. Lemma. If $\left\{\left(R_{n}, S_{n}\right)\right\}$ is a sequence in $\mathscr{D}_{b}^{\prime} \times \mathscr{D}_{a}^{\prime}$ and if $R_{0}=\lim R_{n}$ and $S_{0}=\lim S_{n}$ then $\left\langle\left(R_{0}, S_{0}\right)\right\rangle=\lim \left\langle\left(R_{n}, S_{n}\right)\right\rangle$.

Proof. Let $w \in W$. If $R_{0}=\lim R_{n}$ and $S_{0}=\lim S_{n}$ then

$$
\begin{aligned}
& \left\langle R_{0}(x), \phi(x)\right\rangle=\lim _{n \rightarrow \infty}\left\langle R_{n}(x), \phi(x)\right\rangle \quad(\text { all } \phi \in \mathscr{D}((-\infty, b))), \\
& \left\langle S_{0}(x), \phi(x)\right\rangle=\lim _{n \rightarrow \infty}\left\langle S_{n}(x), \phi(x)\right\rangle \quad(\text { all } \phi \in \mathscr{D}((a, \infty))) .
\end{aligned}
$$

Therefore, for ${ }^{-} 0<t<b$,

$$
\begin{aligned}
& \left\langle\left(R_{0}, S_{0}\right)\right\rangle w(t)=\left\langle R_{0}(x), p(x) w_{t}(x)\right\rangle \\
& \quad=\lim _{n \rightarrow \infty}\left\langle R_{n}(x), p(x) w_{t}(x)\right\rangle=\lim _{n \rightarrow \infty}\left\langle\left(R_{n}, S_{n}\right)\right\rangle w(t) \quad(\text { all } p \in[t]),
\end{aligned}
$$

and, for $a<t<0$,

$$
\begin{aligned}
\left\langle\left(R_{0}, S_{0}\right)\right\rangle w(t) & =-\left\langle S_{0}(x), q(x) w_{t}(x)\right\rangle \\
& =\lim _{n \rightarrow \infty}-\left\langle S_{n}(x), q(x) w_{t}(x)\right\rangle=\lim _{n \rightarrow \infty}\left\langle\left(R_{n^{\prime}} S_{n}\right)\right\rangle w(t) \quad(\text { all } q \in[t]) .
\end{aligned}
$$

2.17. Definition. For any $\phi$ in $\mathscr{D}((-\infty, \infty))$ and any real $t$ we define $\phi_{t}(x)=\phi(t-x)$ for all $x$.

2.18. Theorem. The mapping $(R, S) \mapsto\langle(R, S)$ is a linear bijection of $\mathfrak{D}_{b}^{\prime} \times \mathfrak{D}_{a}^{\prime}$ onto $\mathfrak{Q}$.

Proof. It is easily seen that the mapping is linear. We show first that it is "onto." Let $A \in \mathbb{Q}$ and define

$$
f_{n}(x)=\left\{\begin{array}{ll}
A w_{n}(x) & \text { for } 0 \leq x<b, \\
0 & \text { for } x<0 ;
\end{array} g_{n}(x)= \begin{cases}A w_{n}(x) & \text { for } a<x<0, \\
0 & \text { for } x>0\end{cases}\right.
$$

(see 2.14). Then $\partial^{0} f_{n} \in \mathcal{D}_{b}^{\prime}$ and $\partial^{0} g_{n} \in \mathscr{D}_{a}^{\prime}$. For any $\phi$ in $\mathscr{D}((-\infty, b))$ there exists $t \in(0, b)$ such that $\operatorname{supp} \phi \subset(-\infty, t]$ and therefore $\phi_{t} \in W$. Thus, 


$$
\left\langle\partial^{0} f_{n}(x), \phi(x)\right\rangle=\left\langle\partial^{0} f_{n}(x),\left(\phi_{t}\right) t_{t}(x)\right\rangle=\left\langle f_{n}\right\rangle \phi_{t}(t) .
$$

Combining (1) and 2.14 we have $\lim _{n \rightarrow \infty}\left\langle\partial^{0} f_{n}(x), \phi(x)\right\rangle=A\left(\phi_{t}\right)(t)$. Thus the sequence $\left\{\left\langle\partial^{0} f_{n}(x), \phi(x)\right\rangle\right\}$ converges for all $\phi$ in $\mathscr{D}((-\infty, b))$. By [1, Proposition 2, p. 315] there exists $R$ in $D^{\prime}((-\infty, b))$ such that $R=\lim \partial^{0} f_{n}$; it is easily seen that $R \in \mathscr{D}_{b}^{\prime}$. For any $\phi$ in $\mathscr{T}((a, \infty))$ there exists $t \in(a, 0)$ such that supp $\phi \subset[t, \infty)$ and therefore $\phi_{t} \in W$. Thus,

$$
-\left\langle\partial^{0} g_{n}(x), \phi(x)\right\rangle=-\left\langle\partial^{0} g_{n}(x),\left(\phi_{t}\right)_{t}(x)\right\rangle=\left\langle g_{n}\right\rangle \phi_{t}(t) .
$$

Combining (2) and 2.14 we have $\lim _{n \rightarrow \infty}-\left\langle\partial^{0} g_{n}(x), \phi(x)\right\rangle=A\left(\phi_{t}\right)(t)$. Thus the sequence $\left\{\left\langle\partial^{0} g_{n}(x), \phi(x)\right)\right\}$ converges for all $\phi$ in $\mathscr{L}((a, \infty))$. We may similarly infer the existence of $S$ in $\mathcal{D}_{a}^{\prime}$ such that $S=\lim \partial^{0} g_{n}$. We may now use 2.16, 2.13 and 2.14 to obtain

$$
\begin{aligned}
\langle(R, S)\rangle & =\left\langle\left(\lim _{n \rightarrow \infty} \partial^{0} f_{n}, \lim _{n \rightarrow \infty} \partial^{0} g_{n}\right)\right\rangle \\
& =\lim _{n \rightarrow \infty}\left\langle\left(\partial^{0} f_{n}, \partial^{0} g_{n}\right)\right\rangle=\lim _{n \rightarrow \infty}\left\langle A w_{n}\right\rangle=A ;
\end{aligned}
$$

whence the mapping $(R, S) \mapsto\langle(R, S)\rangle$ is "onto." If $A=\mathbf{0}$ then each $f_{n}$ and each $g_{n}$ equal 0 , from which it follows that $R=\lim _{n \rightarrow \infty} \partial^{0} f_{n}=0$ and $S=\lim _{n \rightarrow \infty} \partial^{0} g_{n}=$ 0 . The mapping $(R, S) \mapsto\langle(R, S)\rangle$ is therefore one-to-one.

2.19. Theorem. The space $\mathfrak{A}$ is sequentially complete.

Proof. Suppose $\left\{A_{n}\right\}$ is a Cauchy sequence in $\mathfrak{Q}$. By 2.17 there exists a unique $\left(R_{n}, S_{n}\right)$ in $\mathscr{D}_{b}^{\prime} \times \mathscr{D}_{a}^{\prime}$ such that $\left\langle\left(R_{n}, S_{n}\right)\right\rangle=A_{n}$ and, by assumption, the sequence $\left\{\left\langle\left(R_{n}, S_{n}\right)\right\rangle w(t)\right\}$ converges for all $w$ in $W$ and all $t \in \Omega$. For any $\phi$ in $\mathcal{D}((-\infty, b))$ there exists $t \in(0, b)$ such that supp $\phi \subset(-\infty, t]$ and therefore $\phi_{t} \in W$. Since $\left\langle R_{n}(x), \phi(x)\right\rangle=\left\langle R_{n}(x), p(x)\left(\phi_{t}\right)(x)\right\rangle=\left\langle\left(R_{n}, S_{n}\right)\right\rangle_{\phi_{t}}(t)$ for all $p \in[t]$, the sequence $\left\{\left\langle R_{n}(x), \phi(x)\right\rangle\right\}$ converges for all $\phi$ in $\mathscr{D}((-\infty, b))$. For any $\phi$ in $\mathcal{D}((a, \infty))$ there exists $t \in(a, 0)$ such that supp $\phi \subset[t, \infty)$ and therefore $\phi_{t} \in W$. Since $-\left\langle S_{n}(x), \phi(x)\right\rangle=-\left\langle S_{n}(x), q(x)\left(\phi_{t}\right),(x)\right\rangle=\left\langle\left(R_{n}, S_{n}\right)\right\rangle \phi_{t}(t)$ the sequence $\left\{\left\langle S_{n}(x), \phi(x)\right\rangle\right\}$ converges for all $\phi$ in $\mathscr{D}((a, \infty))$. We may again use [1, Proposition 2, p. 315] to infer the existence of $(R, S)$ in $\mathscr{D}_{b}^{\prime} \times \mathscr{D}_{a}^{\prime}$ such that $R=\lim R_{n}$ and $S=\lim S_{n}$. By 2.16 we then have

$$
\langle(R, S)\rangle=\lim _{n \rightarrow \infty}\left\langle\left(R_{n}, S_{n}\right)\right\rangle=\lim _{n \rightarrow \infty} A_{n} .
$$

2.20. Lemma. If $(r, s)$ and $(R, S)$ belong to $\mathfrak{T}_{b}^{\prime} \times \mathscr{T}_{a}^{\prime}$ then $\langle(r * R,-s * S)\rangle=$ $\langle(r, s)\rangle\langle(R, s)\rangle$. 
Proof. Let $\left\{\left(F_{n}, b_{n}, J_{n}, G_{n}, a_{n}, K_{n}\right)\right\} \in \Sigma_{R, S}$ and $\left\{\left(f_{n}, b_{n}, j_{n}, g_{n}, a_{n}, k_{n}\right)\right\} \epsilon$ $\Sigma_{r, s^{*}}$ By 1.05 and 1.04,

$$
\begin{gathered}
\left.r * R=\lim _{N \rightarrow \infty} \sum_{m=0}^{N} \sum_{n=0}^{N} \partial^{j}{ }^{+J} n_{m} \wedge F_{n}\right), \\
-s * S=\lim _{N \rightarrow \infty} \sum_{m=0}^{N} \sum_{n=0}^{N} \partial^{k_{m}+K_{n}}\left(g_{m} \wedge G_{n}\right) .
\end{gathered}
$$

Therefore, by 2.16 ,

$$
\begin{aligned}
\langle(r * R,-s * S)\rangle & =\lim _{N \rightarrow \infty} \sum_{m=0}^{N} \sum_{n=0}^{N}\left\langle\left(\partial^{j}{ }^{+J} n\left(f_{m} \wedge F_{n}\right), \partial^{k_{m}+K}{ }_{n}\left(G_{m} \wedge G_{n}\right)\right)\right\rangle \\
& =\lim _{N \rightarrow \infty} \sum_{m=0}^{N} \sum_{n=0}^{N}\left(D^{j_{m}+J}\left\langle f_{m} \wedge F_{n}\right\rangle+D^{k_{m}+K_{n}}\left\langle g_{m} \wedge G_{n}\right\rangle\right) \\
& =\lim _{N \rightarrow \infty} \sum_{m=0}^{N} \sum_{n=0}^{N}\left(D^{j}\left\langle f_{m}\right\rangle D^{J}\left\langle F_{n}\right\rangle+D^{{ }^{k}}{ }^{m}\left\langle g_{m}\right\rangle D^{K}\left\langle G_{n}\right\rangle\right) ;
\end{aligned}
$$

the second equality is from 2.13 and the third equality is from (2.03). Let $w \in W$ and $t \in \Omega$. Choose $N$ sufficiently large so that $a_{N+1}<t<b_{N+1}$. Suppose first that $0 \leq t<b$. Then

$$
\begin{aligned}
\langle(r * R,-s * S)\rangle w(t) & =\sum_{m=0}^{N} \sum_{n=0}^{N} D^{j}\left\langle f_{m}\right\rangle D^{J^{n}}\left\langle F_{n}\right\rangle w(t) \\
& =\sum_{m=0}^{N} D^{j}\left\langle f_{m}\right\rangle\left(\sum_{n=0}^{N} D^{J n}\left\langle F_{n}\right\rangle w\right)(t) \\
& =\sum_{m=0}^{\infty} D^{j}\left\langle f_{m}\right\rangle\left(\sum_{n=0}^{N} D^{J_{n}}\left\langle F_{n}\right\rangle w\right)(t) \\
& \left.=\langle(r, s)\rangle\left(\sum_{n=0}^{N} D^{J_{n}\left\langle F_{n}\right\rangle}\right\rangle\right)(t) ;
\end{aligned}
$$

the last equality is from 2.12. Therefore, by 2.15 and the fact that $\mathcal{Q}$ is a commutative algebra, we have 


$$
\begin{aligned}
\langle(r * R,-s * S)\rangle w(t) & =\sum_{n=0}^{N} D^{J}\left\langle F_{n}\right\rangle(\langle(r, s)\rangle w)(t) \\
& =\sum_{n=0}^{\infty} D^{J}\left\langle F_{n}\right\rangle(\langle(r, s)\rangle w)(t)=\langle(R, s)\rangle(\langle(r, s)\rangle w)(t) \\
& =\langle(R, s)\rangle\langle(r, s)\rangle w(t)=\langle(r, s)\rangle\langle(R, s)\rangle w(t) .
\end{aligned}
$$

And, if $a<t<0$, then

$$
\begin{aligned}
& \langle(r * R,-s * S)\rangle w(t)=\sum_{m=0}^{N} \sum_{n=0}^{N} D^{k} m\left\langle g_{m}\right\rangle D^{K}{ }^{K}\left\langle G_{n}\right\rangle w(t) \\
& \quad=\sum_{m=0}^{N} D^{k}{ }^{m}\left\langle g_{m}\right\rangle\left(\sum_{n=0}^{N} D^{K}\left\langle G_{n}\right\rangle w\right)(t)=\sum_{m=0}^{\infty} D^{k}{ }^{k}\left\langle g_{m}\right\rangle\left(\sum_{n=0}^{N} D^{K}\left\langle G_{n}\right\rangle w\right)(t) \\
& =\langle(r, s)\rangle\left(\sum_{n=0}^{N} D^{K}\left\langle G_{n}\right\rangle w\right)(t)=\sum_{n=0}^{N} D^{K}{ }^{n}\left\langle G_{n}\right\rangle(\langle(r, s)\rangle w)(t) \\
& =\sum_{n=0}^{\infty} D^{K}\left\langle G_{n}\right\rangle(\langle(r, s)\rangle w)(t)=\langle(R, s)\rangle(\langle(r, s)\rangle w)(t)=\langle(r, s)\rangle\langle(R, s)\rangle w(t) .
\end{aligned}
$$

2.21. Definition. For any $F$ in $\mathscr{B}$ we denote the element $\left\langle\left(F_{+}, F_{-}\right)\right\rangle$of $\mathfrak{A}$ by $\langle F\rangle$.

2.22. Theorem. The equation $\left\langle\partial^{0} f\right\rangle=\langle f\rangle$ bolds for all $f$ in $L$.

Proof. Observing that $\left(\partial^{0} f\right)_{+}=\partial^{0} f_{+}$and $\left(\partial^{0} f\right)_{-}=\partial^{0} f_{-}$we may combine 2.21 with 2.13 to obtain the theorem.

2.23. Theorem. The mapping $F \mapsto\langle F\rangle$ is an isomorphism of $\mathbb{B}$ into $\mathbb{Q}$ and the equation $\langle F \wedge G\rangle=\langle F\rangle\langle G\rangle$ bolds for all $F$ and $G$ in $B$.

Proof. The first assertion comes from combining 1.16 and 2.18. As for the second, since $\langle F \wedge G\rangle=\left\langle F_{+} * G_{+}-F_{-} * G_{-}\right\rangle=\left\langle\left(F_{+} * G_{+},-F_{-} * G_{-}\right)\right\rangle$(see 1.18 and 1.19), we may use 2.20 to obtain $\langle F \wedge G\rangle=\left\langle\left(F_{+}, F_{-}\right)\right\rangle\left\langle\left(G_{+}, G_{-}\right)\right\rangle=$ $\langle F\rangle\langle G\rangle$.

\section{BIBLIOGRAPHY}

1. J. Horvath, Topological vector spaces and distributions. Vol. 1, Addison-Wesley, Reading, Mass., 1966. MR 34 \#4863. 
2. H. Kestelman, Modern theories of integration, 2nd ed., Dover, New York, 1960. MR 23 \#A282.

3. G. Krabbe, Operational calculus, Springer-Verlag, New York, 1970.

4. - An algebra of generalized functions on an open interval; two-sided operational calculus, Bull. Amer. Math. Soc. 77 (1971), 78-84; Correction, ibid., 633. MR 42 \#2262; MR 43 \#833.

5. - Initial-value problems involving generalized functions; two-sided operational calculus, Arch. Math. (Basel) (to appear).

6. - A new algebra of distributions; initial-value problems involving Schwartz distributions (to appear).

7. Linear operators and operational calculus. I, Studia Math. 40 (1971), 199-223.

8. H. Shultz, Linear operators and operational calculus. II, Studia Math. 41 (to appear).

9. F. Treves, Topological vector spaces, distributions and kernels, Academic Press, New York, 1967. MR 37 \#726.

10. G. Krabbe, An algebra of generalized functions on an open interval; two-sided operational calculus, Bull. Amer. Math. Soc. 77 (1971), 78-84. MR 42 \#262.

DEPARTMENT OF MATHEMATICS, CALIFORNIA STATE COLLEGE, FULLERTON, CALIFORNIA 92631 